\title{
The Role of Adrenal Corticosterone in Schedule-Induced Wheel Running
}

\author{
WENJUAN LIN,* GEORGE SINGER ${ }^{1}$ AND MICHEAL PAPASAVA \\ *Institute of Psychology, Academia Sinica, Beijing, People's Republic of China \\ Department of Psychology, La Trobe University, Bundoora, Victoria 3083, Australia
}

Received 15 June 1987

\begin{abstract}
LIN, W., G. SINGER AND M. PAPASAVA. The role of adrenal corticosterone in schedule-induced wheel running. PHARMACOL BIOCHEM BEHAV 30(1) 101-106, 1988.-In 3 experiments, the role of adrenal corticosterone in schedule-induced wheel running was investigated. Data from the first experiment showed that bilateral adrenalectomy significantly suppressed schedule-induced wheel running, but did not influence non-scheduled wheel running. Results from a second experiment, where bilateral adrenalectomy with and without replacement of corticosterone was used, showed that the suppressant effect of adrenalectomy on schedule-induced wheel running was completely reversed by replacement of corticosterone. In Experiment 3, plasma corticosterone levels of all experimental rats were determined at the completion of the last test session. The results showed that a circulating level of corticosterone was necessary for the occurrence of schedule-induced wheel running. These findings show that adrenal corticosterone is involved in the regulation of scheduleinduced wheel running.
\end{abstract}

Schedulẹ-induced wheel running

Adrenalectomy

Corticosterone

Rats

IT has been shown that plasma corticosterone levels during one hour schedule-induced drinking or wheel running are about twice as high as those of rats in control conditions given the same number of pellets without a schedule [28]. Since rats under both conditions were at $80 \%$ of their free feeding weight this elevation in corticosterone levels cannot be due to starvation. Various other studies have also demonstrated that schedule-induced behaviour and adrenal corticosterone secretions are interrelated $[3-5,9,26]$. However, the exact role of adrenal corticosterone in schedule-induced behaviour is unknown. Although adrenalectomy and adrenal demedullation have been reported to affect schedule-induced drinking $[6,30]$, the results are not clear cut. The inconsistency of these results has not been explained, but could be due to the use of an inappropriate model, since adrenal secretions are involved in the salt and water balance of the body $[10,27]$. Wheel running may be more appropriate than drinking for determining the effect of adrenal hormones on scheduleinduced behaviour.

Therefore, the present study was undertaken to investigate the effect of adrenal corticosterone variations on schedule-induced wheel running. In the first experiment wheel running of adrenalectomized and sham operated rats with and without a food delivery schedule was compared. In the second experiment the more specific role of glucocorticoids was investigated by comparing wheel running of adrenalectomized rats with or without corticosterone pellet implantation and with or without a food delivery schedule. In Experiment 3 the plasma corticosterone levels of the eight groups of rats used in the previous two experiments were determined at the completion of the last test session. This allowed the monitoring of corticosterone levels under experimental and control conditions.

\section{GENERAL METHOD}

\section{Subjects}

Experimentally naive, male hooded Long-Evans rats with an initial body weight of about $300 \mathrm{~g}$ were used. Rats were housed individually under temperature controlled conditions $\left(22 \pm 1^{\circ} \mathrm{C}\right)$ with a 12 hour light/12 hour dark cycle. After one day acclimatisation to the laboratory, the rats were body weight reduced over a fourteen day period of restricted food intake to $80 \%$ of their free feeding body weight, and were maintained at this weight throughout the experiment.

\section{Apparatus}

The test chamber was made of clear perspex with a stainless steel barred floor, and measured $33.5 \times 28 \times 42 \mathrm{~cm}$. A food cup was located on one end wall of the chamber approximately $3.5 \mathrm{~cm}$ above the floor. Pellet delivery was automatically controlled by standard relay circuitry. Noyes standard formula $45 \mathrm{mg}$ food pellets were used. The chamber was illuminated by a 40 watt globe and ventilation fans provided masking against external sounds. A $26 \mathrm{~cm}$ diameter running wheel was at the rear of the chamber and turned in either clockwise or counter clockwise direction. The number of

${ }^{1}$ Requests for reprints should be addressed to George Singer, Department of Psychology, La Trobe University, Bundoora, Victoria, 3083, Australia. 
wheel revolutions was recorded on a five digit electromechanical counter. Experimental sessions were conducted in eight chambers simultaneously. All experiments were conducted at the same time each day to eliminate possible circadian effects $[2,7,25]$.

\section{Preparations of Corticosterone Pellets}

Corticosterone pellets were produced according to a modified method of Meyer et al. [20]. One hundred mg corticosterone (Sigma Laboratory) and $100 \mathrm{mg}$ cholesterol (Sigma Laboratory) were placed in a $10 \mathrm{ml}$ beaker, melted carefully in a controlled laboratory Heater/Stirrer, and then thoroughly mixed. The molten substance was transferred to a common canning mold $(1 \mathrm{~cm}$ diameter $\times 0.5 \mathrm{~cm}$ deep), where the substance was allowed to cool. After the pellet had cooled and solidified, it was removed from the canning mold. The hardened pellet was a clear, very light golden hue. Two hundred mg pure cholesterol pellets were treated in the same manner as above. The pellets were then individually weighed and trimmed to $100 \mathrm{mg}$ using a scalpel blade.

\section{Surgery}

Bilateral adrenalectomy was performed via the dorsal approach with animals under $60 \mathrm{mg} / \mathrm{kg}$ pentobarbitone sodium anesthesia [29]. The whole adrenal glands, together with surrounding fatty tissue, were removed. Sham adrenalectomy animals underwent the same surgical procedure, but the adrenal gland was left intact. Rats in the adrenalectomized group with replacement of glucocorticoids were adrenalectomized as described, and a pellet consisting of $50 \mathrm{mg}$ of corticosterone combined with $50 \mathrm{mg}$ of cholesterol was implanted subcutaneously in the nape of the neck at the completion of surgery. Rats in the adrenalectomized group with a pellet of $100 \%$ cholesterol served as vehicle implanted controls. At all times, adrenalectomized and adrenalectomized plus pellets rats were maintained on $0.9 \% \mathrm{NaCl}$ solution in place of their normal water supply for sustaining adrenalectomized animals on a long term basis [27]. Animals were allowed a 7 day recovery period prior to testing. Absence of adrenal tissue was confirmed by necropsy and plasma corticosterone levels.

\section{Biochemical Assay}

Plasma corticosterone levels were measured using a modification of the competitive protein binding globulin assay described by Murphy [21]. Horse serum provided the source of binding protein. CBG concentration was at $5 \%$ horse serum level. $\left[{ }^{3} \mathrm{H}\right]$ Corticosterone was the competitively bound steroid and Dextran-charcoal was the absorbent for unbound steroid. The limit of sensitivity was approximately $0.5 \mathrm{ng}$ corticosterone, and the intra- and inter-assay coefficients of variation were $10 \%$ and $13 \%$ respectively.

\section{Statistics}

The data were analysed using a three-way or two-way analysis of variance (ANOVA), simple main effects test and post hoc Newman-Keuls test.

\section{EXPERIMENT 1}

In this experiment, the hypothesis that adrenal activity is necessary for schedule-induced behaviour to occur was tested.

\section{Subjects and Procedure}

Thirty-one rats were used. Sixteen rats received adrenalectomy surgery, eight of these were used as the schedule group (ADX-Sch), the other eight as the nonschedule group (ADX-Nsch). Fifteen rats served as the two sham adrenalectomized groups: of these, 7 served as the schedule group (Sham-Sch), 8 as the non-schedule group (Sham-Nsch). Rats in the schedule group were exposed for 2 hours per day for 14 consecutive days to a FT $120 \mathrm{sec}$ nonreinforcement contingent schedule under which they received on $45 \mathrm{mg}$ pellet each two minutes. The rats in the non-schedule group were given 60 pellets in a single food presentation at the start of each two hour session. Each animal was habituated to the testing chamber for a period of $30 \mathrm{~min}$ before the experiment commenced on the first day of testing.

\section{Results and Discussion}

The mean number of revolutions over the 14 day period for the 4 experimental groups are shown in Fig. 1. A 2 treatment $\times 2$ conditions $\times 14$ days three way ANOVA revealed a highly significant treatment effect, $F(1,27)=21.19$, $p<0.0001$, condition effect, $\mathrm{F}(1,27)=7.04, p<0.05$, and treatment $\times$ condition interaction, $\mathrm{F}(1,27)=9.35, p<0.005$. In order to isolate the source of the treatment $\times$ condition interaction, simple main effects tests were performed. This analysis revealed a significant difference in wheel running between adrenalectomized and sham groups within schedule conditions, $\mathrm{F}(1,28)=22.99, p<0.001$, but not within nonscheduled conditions. Furthermore, while the sham adrenalectomized group exhibited a significant difference in wheel running between scheduled and non-scheduled conditions, $\mathrm{F}(1,28)=8.17, p<0.001$, this difference was not found for the adrenalectomized group.

The ANOVA also showed a significant day effect, $F(13,351)=8.04, p<0.0001$, day $\times$ treatment interaction, $\mathrm{F}(13,351)=1.94, p<0.056$, day $\times$ condition interaction, $\mathrm{F}(13,351)=2.70, p<0.001$, and day $\times$ treatment $\times$ condition interaction, $\mathrm{F}(13,351)=2.13, p<0.05$. Further, every day main effects tests indicated that there were no significant differences in wheel running between adrenalectomized and sham groups within non-scheduled conditions from day 1 to day 14; however, within scheduled conditions, there were significant differences in wheel running between these two groups from day 1 to day 14 [before 7th day, all $\mathrm{F}(1,392) \geqq 7.09, p<0.01$, after 7 th day all $\mathrm{F}(1,392) \geqq 13.52$, $p<0.0001]$. The analyses also showed that there were no significant differences in wheel running between scheduled and non-scheduled conditions within the adrenalectomized group from day 1 to day 14 , although there were significant differences between the two conditions within the sham adrenalectomized group from day 7 to day 14 [in all cases $\mathrm{F}(1,392) \geqq 6.05, p<0.05]$.

Collectively, these data show that adrenalectomy significantly suppressed schedule-induced wheel running from the first day of testing and that this effect was maintained throughout the 14 day period.

This suppressant effect was not due to a reduced level of activity [8] since non-scheduled wheel running was not significantly affected by adrenalectomy. It was also not due to a possible reduction in food intake, as both adrenalectomized and sham operated rats consumed all schedule-delivered pellets and their daily food rations for maintaining $80 \%$ of their initial body weights. 


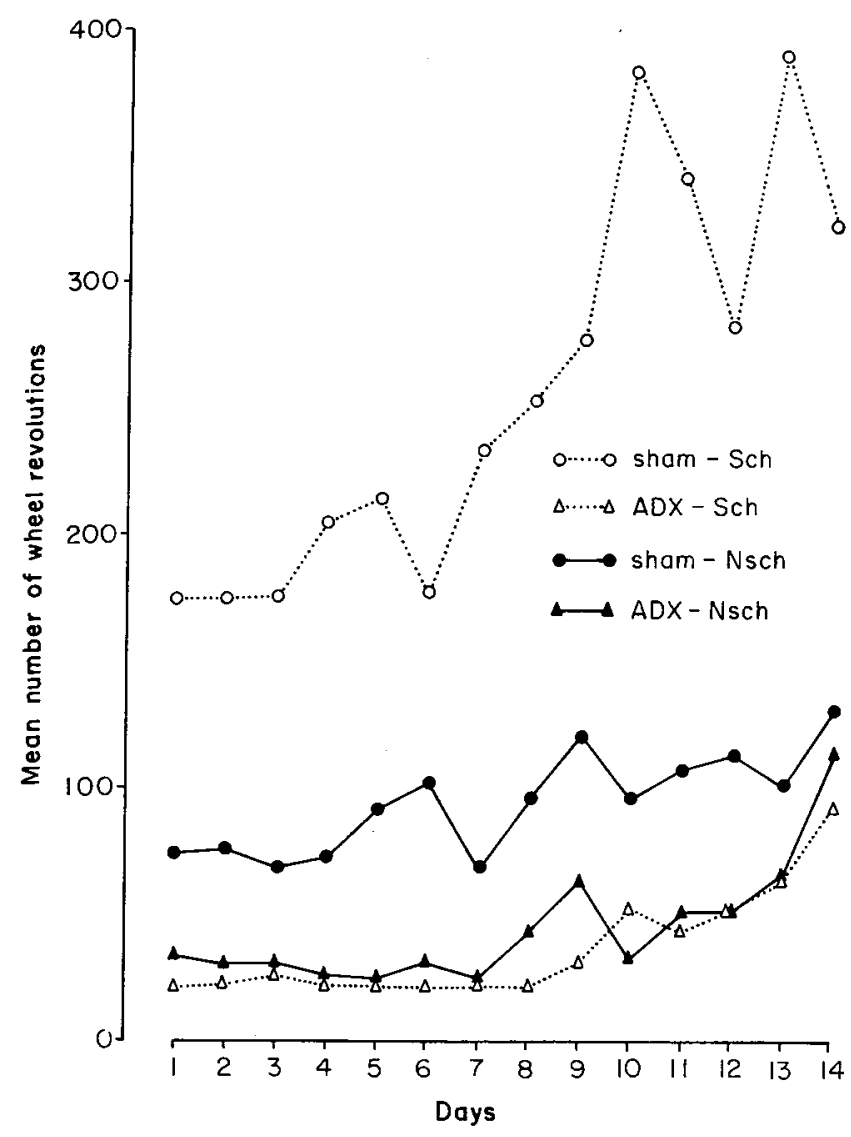

FIG. 1. Mean number of wheel revolutions during two hours of exposure to schedule or non-schedule food for 4 groups of adrenalectomized and sham operated rats.

For the sham-scheduled group, wheel running increased over the 14 day period, but the significant increase, when compared with the non-scheduled group, was from the 7 th day of testing. The former result is consistent with previous work $[15,17,19]$ and the latter suggests that an effective testing of schedule-induced wheel running should last for 7 days or longer.

\section{EXPERIMENT 2}

The results obtained in Experiment 1 indicate that adrenalectomy suppresses schedule-induced wheel running. Although adrenalectomy involves removal of medullary as well as cortical tissue, the glucocorticoids, which are secreted by cortical tissue, are probably the major hormonal influence on this behaviour. Complete bilateral adrenalectomy with and without replacement of the major glucocorticoid should therefore adequately test this hypothesis.

\section{Subjects and Procedure}

Twenty-six rats were used. All animals were adrenalectomized. Thirteen rats received corticosterone pellets (ADX(C)). Of these, seven rats served as the scheduled group (ADX(C)-Sch), six rats as the non-scheduled group (ADX(C)-Nsch). Another thirteen rats received cholesterol pellets as vehicle implantation. Six of these were used as the scheduled group (ADX (O)-Sch), while seven served as the non-scheduled group (ADX(O)-Nsch). All experiment methods and procedures were the same as for Experiment 1. Two rats in ADX(O)-Sch group died on the last day of testing.

\section{Results and Discussion}

The mean number of revolutions over the 14 day period for the 4 adrenalectomized groups with and without replacement of corticosterone are shown in Fig. 2. The same $2 \times 2 \times 14$ three way ANOVA as used in Experiment 1 showed a significant treatment effect, $\mathrm{F}(1,22)=9.72, p<0.005$, and condition effect, $\mathrm{F}(1,22)=5.56, p<0.05$. The treatment $\times$ condition interaction almost reached a significant level, $\mathrm{F}(1,22)=3.94, p<0.06$. Further, simple main effects tests indicated that there was a significant difference between $\operatorname{ADX}(\mathrm{C})$ and $\mathrm{ADX}(\mathrm{O})$ groups within schedule conditions, $F(1,23)=10.95, p<0.005$, although there was no significant difference between these two groups within non-scheduled conditions. The tests also showed that there was only a significant difference in wheel running between schedule and non-schedule conditions within the $\operatorname{ADX}(C)$ group, $\mathrm{F}(1,23)=7.89, p<0.01$, and no difference between these two conditions within the $\operatorname{ADX}(\mathrm{O})$ group. These tests indicated that only $\operatorname{ADX}(C)-S c h$ rats showed significantly increased wheel running over the 14 day period. Thus, the replacement of corticosterone reversed the suppressant effect of adrenalectomy on schedule-induced wheel running.

The ANOVA also showed a significant day effect, $\mathrm{F}(13,284)=5.14, p<0.0001$, a significant day $\times$ treatment interaction, $F(13,284)=2.42, p<0.005$; day $\times$ condition interaction, $\mathrm{F}(13,284)=2.53, p<0.005$; and day $\times$ treatment $\times$ condition interaction, $F(13,284)=1.92, p<0.05$. Further, simple main effects tests on the day data showed that there were significant differences in wheel running between $\operatorname{ADX}(\mathrm{C})$ and $\mathrm{ADX}(\mathrm{O})$ groups within scheduled conditions from day 3 to day 14 , in all cases $\mathrm{F}(1,333) \geqq 4.81, p<0.05$, but that there were no significant differences in wheel running between $\operatorname{ADX}(C)$ and $\operatorname{ADX}(\mathrm{O})$ groups within non-scheduled conditions from day 1 to day 14 . Furthermore, there were no significant differences between scheduled and nonscheduled conditions within the $\mathrm{ADX}(\mathrm{O})$ group from day 1 to day 14 , however, there were significant differences between these two conditions within the ADX(C) group from day 3 to day 14 , except $p<0.07$ in day 7 , in all other cases, $\mathrm{F}(1,33) \geqq 4.38, p<0.05$.

These findings show that the reversal caused by the replacement of corticosterone occurred on day 3 of testing and persisted throughout almost the entire 14 day period of testing. Thus, corticosterone replacement completely reverses the suppressant effect of adrenalectomy on scheduleinduced wheel running.

In order to study further the relations among all eight groups in Experiment 1 and Experiment 2, a multiple range test at the 0.05 level of significance was performed using the mean wheel revolutions across the 14 days of testing. The analysis indicated that the mean revolutions did not differ from each other among 6 groups (i.e., ADX-Sch, ADX(O)Sch, ADX-Nsch, Sham-Nsch, ADX(O)-Nsch, ADX(C)Nsch). Both Sham-Sch and ADX(C)-Sch rats significantly increased wheel running as compared to any of the other 6 groups. There was, however, no significant difference between these two groups. Another multiple range test at the 0.05 level of significance, using the mean wheel revolutions of the final 4 days, showed the same result. These range tests confirmed that adrenalectomized rats which had corticoste- 


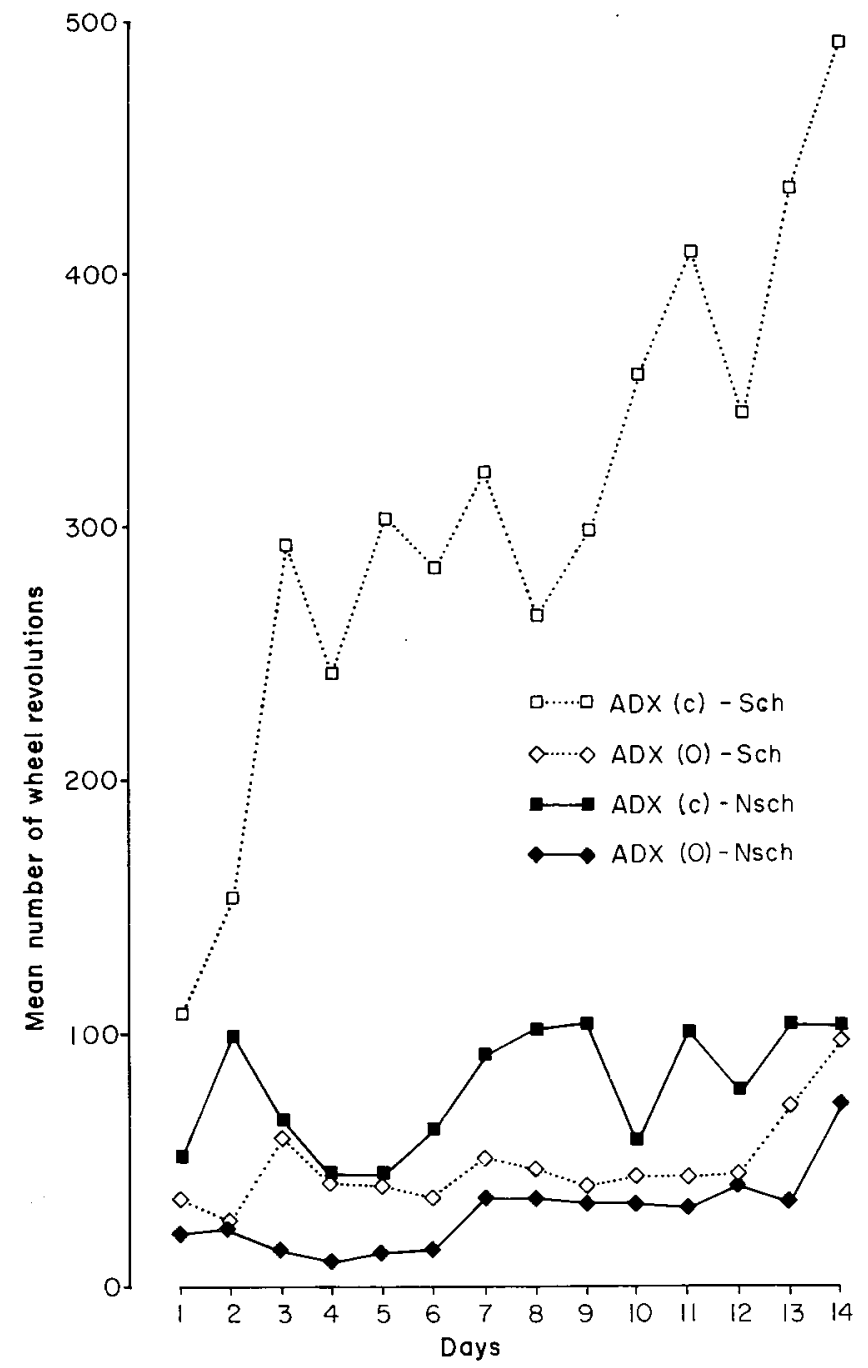

FIG. 2. Mean number of wheel revolutions during two hours of exposure to schedule or non-schedule food for 4 groups of adrenalectomized rats with and without replacement of corticosterone.

rone replaced displayed the same level of wheel running during the 14 day testing priod as the sham adrenalectomized rats. Thus, the implantation of a pellet consisting of $50 \mathrm{mg}$ corticosterone combined with $50 \mathrm{mg}$ cholesterol was fully effective in reinstating schedule-induced wheel running, whereas pure cholesterol had no significant effect upon schedule-induced wheel running.

\section{EXPERIMENT 3}

The data from Experiments 1 and 2 suggested that corticosterone was the major hormonal influence on scheduleinduced wheel running. In Experiment 3, plasma corticosterone levels were determined in order to confirm the role of corticosterone in schedule-induced wheel running.

\section{Subjects and Procedure}

All subjects in Experiments 1 and 2 were sacrificed consecutively by decapitation at the completion of fourteen test sessions. Trunk blood was collected in heparinized tubes and centrifuged immediately at $2200 \mathrm{rpm}$ for 20 minutes. The plasma was stored at $-80^{\circ} \mathrm{C}$ until assayed.

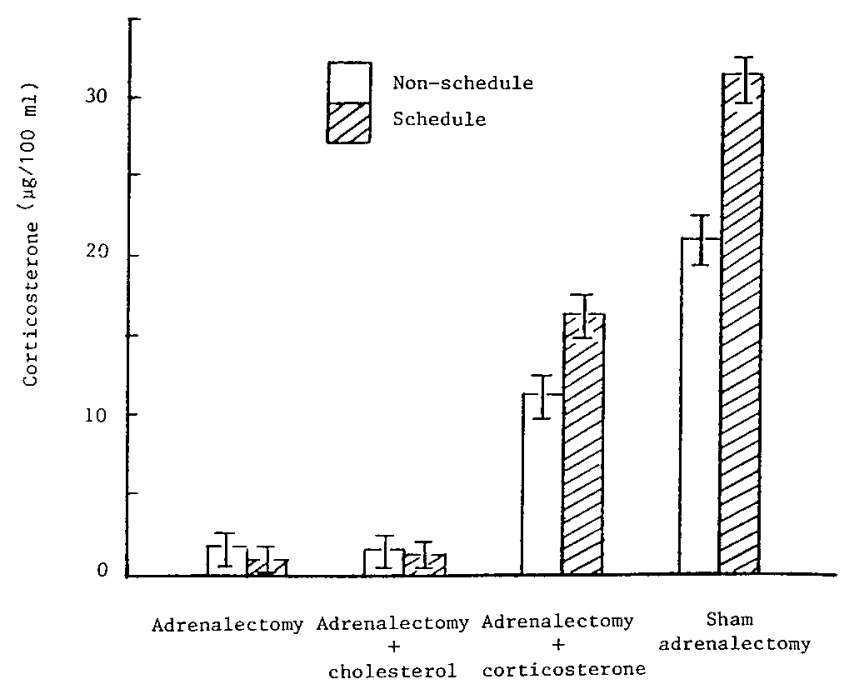

FIG. 3. Mean plasma corticosterone levels (mean $\pm \mathrm{SE})(\mu \mathrm{g} / 100 \mathrm{ml})$ for 8 groups after 14 daily two hour wheel running tests.

\section{Results and Discussion}

Means and standard deviations of plasma corticosterone levels $(\mu \mathrm{g} / 100 \mathrm{ml})$ are given in Fig. 3. A two way ANOVA showed significant treatment effect, $F(3,47)=88.27$, $p<0.0001$, condition effect, $\mathbf{F}(1,47)=6.97, p>0.05$, and treatment $\times$ condition interaction, $\mathrm{F}(3,47)=5.86, p<0.005 . \mathrm{A}$ post hoc Newman-Keuls test at the 0.05 level of significance showed that all adrenalectomized and adrenalectomized with vehicle implantation groups had significantly lower levels than sham adrenalectomized or adrenalectomized with corticosterone pellet groups, but did not differ from each other significantly. Sham groups had significantly higher levels of corticosterone than adrenalectomized with corticosterone pellet groups, regardless of scheduled or non-scheduled conditions. However, sham group within the schedule condition had significantly higher levels of corticosterone than did sham group within the non-scheduled condition, while adrenalectomized with corticosterone pellet groups did not differ significantly from each other within the conditions.

The corticosterone values detected in the adrenalectomized and adrenalectomized with vehicle implanted rats were extremely low, confirming total adrenalectomy. The sham adrenalectomy group showed relatively high levels of corticosterone, and the increase in corticosterone levels of the sham-scheduled group, compared to the non-scheduled group was in accordance with previous work [9, 26, 28] and might be a response to schedule-induced stress. The adrenalectomized rats with corticosterone pellets showed intermediate corticosterone levels which were nonresponsive to schedule-induced stress.

\section{GENERAL DISCUSSION}

The present findings suggest that adrenal corticosterone plays a major role in schedule-induced wheel running. The results of Experiment 1 indicate that adrenalectomy significantly suppressed wheel running under scheduled conditions while it did not significantly affect wheel running under nonscheduled conditions. The data from Experiment 2 suggest that corticosterone which was secreted from the adrenal cortex was the major influence on schedule-induced wheel running, as corticosterone replacement through implantation virtually reversed the suppressant effect of adrenalectomy 
on schedule-induced wheel running. Finally, the data from Experiment 3 show quantitatively that a given level of plasma corticosterone was necessary for the occurrence of schedule-induced wheel running.

Since almost any kind of stress to which the animal is subjected can cause secretion of adrenal cortical hormones $[1,11,12,16,22,24]$ plasma corticosterone levels are frequently used as an index of stress or arousal in studies of schedule-induced behaviour $[5,9,26,28]$. Corticosterone elevations, as suggested by previous work [9], may be a concomitant of schedule-induced behaviour. The increase of plasma corticosterone levels in sham operated rats within scheduled conditions supports this argument. Moreover the present findings suggest that corticosterone itself controls the occurrence of schedule-induced behaviour. Therefore it has been suggested that corticosterone is not only a concomitant secretion to schedule-induced behaviour but also is involved in the acquisition and maintenance of scheduleinduced behaviour.

It is of interest to note that although plasma corticosterone levels in adrenalectomized rats with corticosterone replacement were still significantly lower than in sham operated rats, the schedule-induced behaviour suppressed by adrenalectomy was completely reversed at this level. There are at least two possible explanations for this finding. First, adrenal corticosterone exerts a permissive action whereby animals can exhibit adjunctive behaviour under scheduled conditions only in the presence of certain small amounts of corticosterone. Second, the binding capacity of receptors or the amount of binding protein to corticosterone in the brain is increased by adrenalectomy $[18,23]$, thus the exogenous corticosterone in adrenalectomized rats may be more effective than the same level of endogenous corticosterone in intact rats.

It seems that the present findings cannot be compared with the work of Devenport [6] or of Wright and Kelso [30] since the schedule-induced behaviour models are different. However, it should be noted that although adrenalectomy may facilitate the onset of schedule-induced drinking as Devenport has reported, this does not mean that adrenalectomy cannot attenuate or depress the development of scheduleinduced drinking when such animals are maintained on a daily scheduled condition for a period of time. In fact, it has been shown that there is an abrupt decline in scheduleinduced drinking following adrenalectomy when animals are placed on a 90 pellets $/ 90$ min session for a period of 30 days [30]. Wright and Kelso attempted to demonstrate that the suppressant effect of adrenalectomy on schedule-induced drinking was due to adrenal medulla, not cortical, mechanisms. However, the fact that pharmacological demedullation failed to suppress schedule-induced drinking (SID) while surgical demedullation influenced SID indicates that the role of the adrenal medulla in schedule-induced drinking needs further examination and the role of the adrenal cortex cannot be excluded.

Recently, it has been shown that a behavioural effect of adrenalectomy can be reversed by both glucocorticoids and by [D-Ala-Met $\left.{ }^{5}\right]$ enkephalinamide $[13,14]$, suggesting that either adrenocortical glucocorticoids or adrenomedullary peptides are involved in modulating some kinds of behaviour. Therefore, it is of interest to determine whether corticosterone is uniquely necessary for the occurrence of schedule-induced wheel running, or whether adrenomedullary secretions also modulate schedule-induced wheel running in the absence of adrenal corticosterone. In addition, it is also necessary to provide evidence which shows that the effects of corticosterone on schedule-induced behaviour are not due simply to the secondary actions of adrenalectomy increasing ACTH secretions [19]. Further research is currently in progress.

\section{REFERENCES}

1. Ader, R. and S. B. Friedman. Plasma corticosterone response to environmental stimulation. Effects of duration of stimulation and 24-hour adrenocortical rhythm. Neuroendocrinology 3: 378-386, 1968.

2. Barnett, S. A. The Rat: A Study in Behaviour, revised edition. Canberra: Australian National University Press, 1976, pp. 37-38.

3. Brett, L. P. and S. Levine. Schedule-induced polydipsia suppresses pituitary-adrenal activity in rats. J Comp Physiol Psychol 93: 946-956, 1979.

4. Brett, L. P. and S. Levine. The pituary-adrenal response to "minimized" scheduled-induced drinking. Physiol Behav 26: 153-158, 1981.

5. Dantzer, R. and P. Mormede. Pituitary-adrenal consequences of adjuctive activities in pigs. Horm Behav 15: 386-395, 1981.

6. Devenport, L. D. Schedule-induced polydipsia in rats: Adrenocortical and hippocampal modulation. I Comp Physiol Psychol 92: 651-660, 1978.

7. Dunn, J., L. Scheving and P. Millet. Circadian variation in stress-evoked increase in plasma corticosterone. Am J Physiol 223: 402-406, 1972.

8. Durrant, E. P. Studies in vigor. Effect of adrenal extirpation on activity of the albino rat. Am J Physiol 70: 344-350, 1924.

9. Finlay, J. D. and M. Wallace. Effect of scheduled food delivery on corticosterone levels in the rat. Proc Aust Neurosci Soc 1: $81,1981$.

10. Frieden, E. H. Chemical Endocrinology. New York: Academic Press, 1976, pp. 2-5.
11. Galina, Z. H., C. J. Sutherland and Z. Amit. Effects of heatstress on behaviour and pituitary adrenal axis in rats. Pharmacol Biochem Behav 19: 251-256, 1983.

12. Hennessy, M. B. and S. Levine. Sensitive pituitary-adrenal responsiveness to varying intensities of psychological stimulation. Physiol Behav 21: 295-297, 1978.

13. Jefferys, D., D. Copolov, D. Irby and J. W. Funder. Behaviour effect of adrenalectomy: Reversal by glucocorticoids of [D$\mathrm{Ala}^{2}, \mathrm{Met}^{5}$ ] enkephalinamide. Eur J Pharmacol 92: 99-104, 1983.

14. Jefferys, D., D. Copolov and J. W. Funder. Naloxone inhibits both glucocorticoid and [D-Ala, Met $^{5}$ ] enkephalinamide reversal of behavioural effect of adrenalectomy. Eur $I$ Pharmacol 103: 205-210, 1984.

15. King, G. D. Wheel running in the rat induced by a fixed-time presentation of water. Anim Learn Behav 21: 325-328, 1974.

16. Knigge, K. M., C. H. Period and J. Schindler. In vitro and vivo adrenal corticosterone secretion following stress. Am J Physiol 196: 579-582, 1959.

17. Levitsky, D. and G. Collier. Schedule-induced wheel running. Physiol Behav 3: 571-573, 1968.

18. McEwan, B. S., G. Wallach and C. Magnus. Corticosterone binding to hippocampus: immediate and delayed influence of the absence of adrenal sections. Brain Res 70: 321-334, 1974.

19. Matsuyama, H., R. B. Mins, A. Ruhmann-Wennhold and D. H. Nelson. Bioassay and radioimmunoassay of plasma ACTH in adrenalectomized rats. Endocrinology 88: 696-701, 1971.

20. Meyer, J. S., D. J. Micco, B. S. Stephenson, L. C. Krey and B. S. McEwan. Subcutaneous implantation method for chronic glucocorticoid replacement therapy. Physiol Behav 22: 867-870, 1979. 
21. Person-Murphy, B. E. Some studies of the protein-binding of steroids and their application to the routine micro and ultramicro measurement of various steroids in body fluids by competitive protein-binding radioassay. J Clin Endocrinol 27: 973990, 1967.

22. Pfister, H. P. The glucocorticosterone response to novelty as a psychological stressor. Physiol Behav 23: 649-652, 1979.

23. Stevens, W., D. J. Reed, S. Erickson and B. I. Grosser. The binding of corticosterone to brain proteins: Diurnal variation. Endocrinology 93: 1152-1156, 1973.

24. Stockham, M. A. Changes of plasma and adrenal corticosterone levels in the rat after repeated stimuli. J Physiol 193: 149-159, 1964.

25. Takahashi, K., K. Inoue and Y. Takahashi. Parallel shift in circadian rhythms of adrenocortical activity and food intake in blinded and intact rats exposed to continuous illumination. Endocrinology 100: 1097-1107, 1977.
26. Tang, C., M. Wallace, G. Singer and L. Mackenzie. Resistance of schedule-induced behaviours to hippocampal lesions. Pharmacol Biochem Behav 20: 527-541, 1984.

27. Turner, C. D. and J. T. Bagnara. General Endocrinology. Philadelphia: W. B. Saunders Company, 1976, pp. 571-376.

28. Wallace, M., G. Singer, J. Finlay and S. Gibson. The effect of 6-OHDA lesions of the nucleus accumbens septum on schedule-induced drinking, wheel running and corticosterone levels in the rat. Pharmacol Biochem Behav 18: 129-136, 1983.

29. Waynforth, H. B. Experimental and Surgical Technique in the Rat. New York: Academic Press, 1980, pp. 124-127.

30. Wright, J. W. and S. C. Kelso. Adrenal demedullation suppresses schedule-induced polydipsia in rats. Physiol Behav 26: 1-5, 1981 . 